\title{
Developmental differences in recall and output organization
}

\author{
PETER A. ORNSTEIN \\ University of North Carolina, Chapel Hill, North Carolina 27514 \\ and \\ GORDON A. HALE and JUDITH S. MORGAN \\ Educational Testing Service, Princeton, New Jersey 08540
}

\begin{abstract}
Third- and seventh-grade children were given a free recall task either with unrelated pictures or with pictures representing members of common categories. Mean number of items recalled increased with age for both related and unrelated lists. However, whereas the tendency to cluster related items increased with age, use of subjective organization with unrelated materials did not increase. The lack of developmental change in subjective organization was observed not only with Bousfield and Bousfield's (1966) traditional index of pairwise consistencies, but also with Pellegrino's (1971) measures of higher order organization. The data suggest that developmental increases in the amount of information recalled are not solely a function of age differences in amount of output organization.
\end{abstract}

Although developmental improvement in free recall has often been demonstrated, the relation between children's recall and organizational activities is unclear. Recall improvement is observed both with materials drawn from common taxonomic categories and with relatively unrelated items. However, even though a recent review (Jablonski, 1974) has indicated otherwise, the existing literature does suggest that developmental patterns in measured output organization differ in these two situations. These data imply that different factors may be responsible for age-related improvement in the recall of related and unrelated lists.

With taxonomically related items, category clustering (Bousfield, 1953; Bousfield \& Bousfield, 1966), reflecting the extent to which recall is structured according to the categories provided by the experimenter, increases with age (Bousfield, Esterson, \& Whitmarsh, 1958; Cole, Frankel, \& Sharp, 1971). The corresponding increases in recall and output organization with these materials are consistent with the view that developmental changes in organization may be responsible for the observed recall improvement. However, with unrelated items, subjective organization (Bousfield \& Bousfield, 1966; Tulving, 1962), reflecting the imposition of a personal structure upon the materials, does not

This work was supported by Grant OEG-2-9-4004431060(010) from the Office of Education. Preparation of this report was facilitated by Grant HD 08459 from the National Institute of Child Health and Human Development. Appreciation is expressed to officials at the Council Rock School System in Bucks County, Pennsylvania for their assistance in obtaining subjects. Thanks are also due Miriam Vaughan for making available copies of her pictorial materials, some of which were employed here, and David F. Bjorklund for comments on an earlier draft of this report. appear to change substantially over a considerable age range. Thus, with children ranging from approximately 5.5 to 10.5 years, Laurence (1966) observed clear age differences in recall, but none in subjective organization. Although the recall and subjective organization of Laurence's college-age subjects was greater than that of the children, the children's data cast doubt upon the role of subjective organization as a mediator of developmental changes in the recall of unrelated items.

Similar findings of recall differences but equivalent subjective organization have been reported by Nelson (1969), with 5- and 8-year-old children; Shapiro and Moely (1971), with subjects ranging between 9 and 13 years; and Ornstein, Naus, and Liberty (1975, Experiment 1 ), with children between 8.5 and 13.5 years. Kokubun (1973), working with 8-, 11-, and 14-yearolds, reported no differences in measured subjective organization until the final trials of a 16-trial sequence. However, since inspection of Kokubun's presented data indicates age differences in recall in the early trials, without corresponding differences in organization, it is difficult to view subjective organization as a critical factor in accounting for the recall differences. Only one study (Rosner, 1971), reported significant evidence of an age difference in children's subjective organization. The recall and subjective organization of Rosner's first graders (under standard conditions) were less than that of the fifth- and ninth-grade subjects (although these two groups of older subjects had equivalent organization but different levels of recall).

The studies cited above do not appear to support Jablonski's (1974) claim that organization increases with age for unrelated as well as related materials. Nonetheless, a strong conclusion that there is little developmental change in organization with unrelated materials 
presupposes that a comprehensive search for such organization has been undertaken. In fact, the indices of subjective organization employed in the abovementioned studies examine the recall protocols only for pairs of items recalled contiguously on adjacent trials; these measures may thus underestimate the presence of higher order subjective organization. Given these possible measurement problems, the purpose of the present study was to re-examine the relationship between developmental changes in output organization (both clustering and subjective organization) and recall. To search for possible subjective organization, Pellegrino's (1971) indices, which measure not only intertrial repetition of adjacent items, but also higher order consistencies, were employed. Developmental changes from ages 9 to 13 were examined since previous studies generally have found no change over this period in children's subjective organization but a marked improvement in recall.

\section{METHOD}

\section{Design and Materials}

At each of two age levels (third and seventh grades), two groups of subjects were presented with 25 to-be-remembered items for five alternating study and recall trials. Each of the items was a simple line drawing on a white background corresponding to an object familiar to the children. The two groups differed only in the type of materials they were asked to learn. For one group at each age level, the materials were taxonomically related items, while for the second group, unrelated items were employed. The 25 items on the related list were taken from five taxonomic categories, each with five items; no obvious conceptual relations were present among the 25 items on the unrelated list. To partially control for list-specific effects, two different related lists and two different unrelated lists were constructed, and one-half of the subjects in each group were assigned to each of the alternate lists. The related and unrelated lists were matched with respect to Thorndike-Lorge (1944) frequency of the words depicted.

\section{Procedure}

All subjects were tested individually and were seated at a table opposite a female experimenter. The instructions to the subjects indicated that they would be asked to remember some pictures but did not stress the type of materials which would be presented. On the first presentation trial, subjects were asked to label the stimulus pictures and were corrected by the experimenter when necessary; corrections were rare, and subjects were allowed to use their own labels when synonymous with those of the experimenter (e.g., sofa, couch). The experimenter held up each of the drawings for $4 \mathrm{sec}$. Recall was oral, with
$90 \mathrm{sec}$ allowed for each of the five recall trials, and subjects were free to recall the items in any order. For each set of materials, five different random orders of stimulus presentation, corresponding to the five trials, were generated. These orders were arranged into four different sequences, and one male and one female in each subgroup were exposed to each sequence.

\section{Subjects}

A total of 64 children, 32 at each of the third (mean age $=9.0$ years) and seventh (mean age $=13.0$ years) grades, were randomly assigned to the experimental groups. All subjects came from a middle-class area in Bucks County, Pennsylvania. At each age level, there were 16 subjects in each major group ( 8 boys and 8 girls), one-half of whom learned each set of materials.

\section{RESULTS}

\section{Recall}

The mean number of words recalled, as a function of trials, is shown in Panel A of Figure 1. The performance of the seventh graders was superior to that of the third graders, and children at both age levels showed free recall learning, that is, an increase in recall over trials. Further, at each grade, the subjects learning lists of related items performed better than those learning unrelated items. An analysis of variance applied to these data confirmed visual inspection of the curves. There were significant effects of Age $[F(1,60)=23.95$, $\mathrm{p}<.001]$, Type of Lists (related vs. unrelated) $[\mathrm{F}(1,60)$ $=51.94, \mathrm{p}<.001]$, and Trials $[\mathrm{F}(4,240)=78.79$, $p<.001]$. Although the difference between the two age groups seems somewhat greater with the related materials than with the unrelated items, the Age by Type of List interaction was not significant $[\mathrm{F}(1,60)$ $=1.62, \mathrm{p}<.25]$.

\section{Organization}

To explore organizational activities which might underlie the superior recall of the older subjects with both sets of materials, as well as the over-trials improvement in recall for all subjects, categorical clustering scores and subjective organization measures were computed. The clustering scores, measured by Bousfield and Bousfield's (1966) stimulus category repetition index, for the related item conditions are plotted in Panel B of Figure 1. The O(SCR) - E(SCR) measure represents a count of the number of times an item from one category is followed in recall by another member
Figure 1. Mean number of words recalled (Panel A) as a function of trials for third and seventh graders given related and unrelated materials, mean O(SCR) - E(SCR) scores (Panel B) as a function of trials for the related item conditions, and mean O(ITR) - E(ITR) scores (Panel C) as a function of trial pairs for the unrelated item conditions.
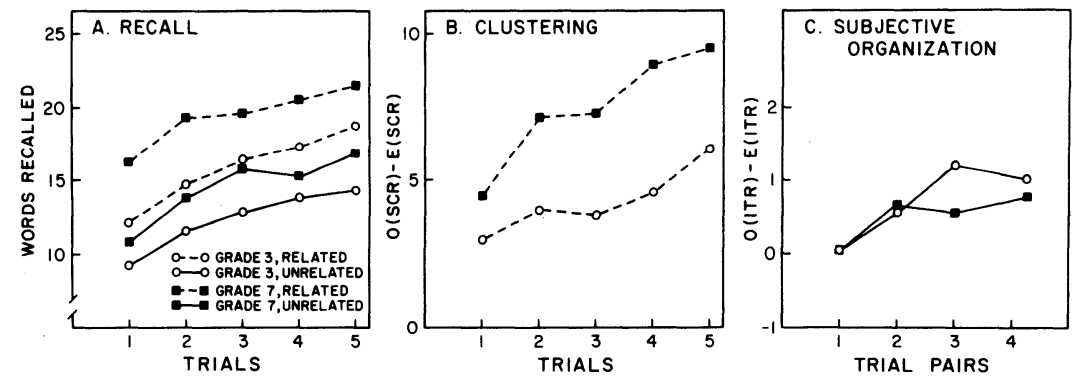
of the same category; the observed repetition value is compared with a chance value. Inspection of Panel B indicates that group differences in degree of clustering parallel the recall data. Clustering increased with both Age $[F(1,30)=18.62, p<.001]$ and Trials $[F(4,120)=$ $18.08, \mathrm{p}<.001]$. The divergence of the two curves is reflected in a marginal Age by Trials interaction $[F(4,120)=2.22, p<.10]$.

Subjective organization, measured in terms of Bousfield and Bousfield's (1966) intertrial repetition index, is plotted in Panel C of Figure 1, for the groups receiving unrelated materials. The O(ITR)-E(ITR) measure calculates the number of pairs of words which were recalled in sequence on each pair of successive trials; the observed repetition value is then compared with a chance value. Inspection of Panel $\mathrm{C}$ indicates that subjective organization increased slightly over trials, but that there were no differences between the two age groups; indeed, the only significant effect in the analysis of variance performed on these data was that due to Trial Pairs $[\mathrm{F}(3,90)=3.58, \mathrm{p}<.05]$.

Since the O(ITR) - E(ITR) measure only concentrates on pairwise contingencies between items recalled on consecutive trials, it is possible that organization actually present in the recall protocols may be underestimated. Pellegrino (1971) has recently developed measures which can examine pairs of trials for groups of words which are larger than Size 2 (e.g., triplets, quartets, etc.). Further, the items need not be recalled in the same order on both trials, but can be recalled in reverse order, or even in an unordered pattern in which any permutation of the items is accepted as a repetition. These different criteria (forward, reverse, and unordered) lead to different expected values which are subtracted from the obtained intertrial repetition, O(ITR), score. A ratio between O(ITR) - E(ITR) and $\operatorname{Max}(I T R)-E(I T R)$ is formed to indicate the proportion of the possible (i.e., above chance) organization present. The obtained ratios are termed ARC' scores, modeled after the adjusted ratio of clustering measure of Roenker, Thompson, and Brown (1971). These ARC' $^{\prime}$ indices were calculated here for the subjects learning unrelated words and are plotted in Figure 2; Panels A, B, and C present the $\mathrm{ARC}^{\prime}$ scores for units of Size 2, 3, and 4, respectively, all measured against the most liberal unordered criterion. These data are noteworthy for several reasons. First, the organization values are very low, expecially for units greater than Size 2; there simply is little higher order organization present in these recall protocols. Second, in contrast to the Bousfield and Bousfield (1966) measure, there are no Trials effects $\left[F_{s}(3,90) \leqslant 1.01\right]$. Third, and most important, is the fact that under no condition is there an age improvement in measured output organization; indeed, the third graders actually showed greater subjective organization than the seventh graders for units of Size $2[F(1,30)=3.93, p<.10]$.

\section{DISCUSSION}

As expected, recall performance increased with age for both types of items. The lack of an interaction of Age by Type of Lists (related vs. unrelated items) is consistent with previous findings (Cole et al., 1971), and indicates comparable improvement with age in the recall of both related and unrelated items, even though the difference between the two grade levels was somewhat greater with the related items. However, although there was age-related improvement in the recall of both classes of items, this improvement seems to be associated with increasing use of organization in the case of the related materials but not with the unrelated items. That is, with materials which were matched iny terms of Thorndike-Lorge (1944) frequency, category clustering increased with age, but subjective organization was constant over the two ages examined. Thus, the data suggest that different factors may be responsible for developmental improvement with these two types of materials.

It seems unlikely that the absence of a developmental change in subjective organization reflects insensitive measurement procedures, since differences were not obtained either with the Bousfield or the Pellegrino indices. It had been thought that, with these more sensitive measures, age differences in higher order subjective units might be detected. However, very little higher order organization was present in the recall protocols, and it is reasonable to conclude that there are no significant differences between the two age groups in subjective organization. Although it could be argued that differences in higher order subjective units might have been obtained if more trials had been included, the possible emergence of such organization on later trials could not account for the recall superiority of the older subjects on the early trials.

Assuming that the developmental change in category clustering contributes to the improvement in the recall of taxonomic materials with age, these data raise a fundamental question as to what accounts for developmental improvement in the recall of unrelated items. Is the amount of measured output organization relatively unimportant in accounting for age differences in the recall of these materials? If so, there still may be untapped differences in the type, if not the amount, of subjective organization which are related to recall performance. In addition, perhaps more direct measures of children's organizational activities at stimulus input (see Liberty \& Ornstein, 1973) will be related to developmental differences in memory. These measures may be developmentally sensitive, but with respect to amount of output organization at least, the present data support the view that age differences in organization cannot fully account for the observed developmental changes in recall.
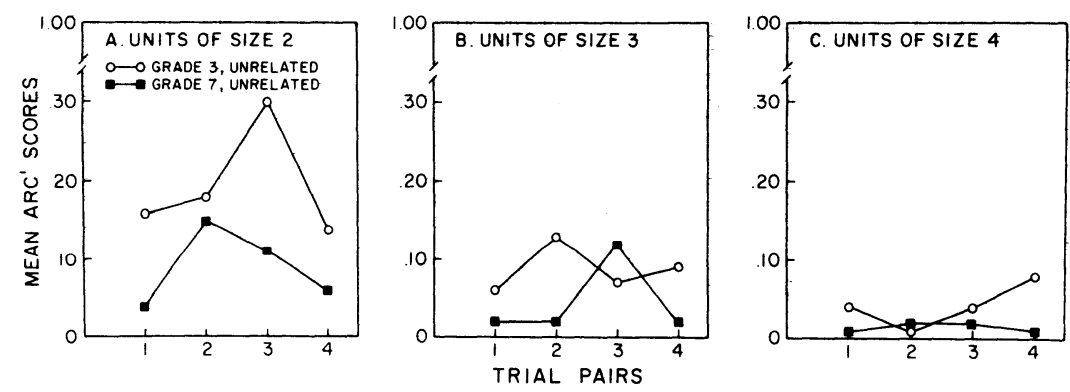

Figure 2. Mean ARC' scores as a function of trial pairs for subjective units of Size 2 (Panel A), Size 3 (Panel B), and Size 4 (Panel C) for third and seventh graders given unrelated materials. 


\section{REFERENCES}

Bousfield, A. K., \& Bousfield, W. A. Measurement of clustering and of sequential constancies in repeated free recall. Psychological Reports, 1966, 19, 935-942.

Bousfield, W. A. The occurrence of clustering in the recall of randomly arranged associates. Journal of General Psychology, 1953, 49, 229-240.

Bousfield, W. A., Esterson, J., \& Whitmarsh, G. A. A study of developmental changes in conceptual and perceptual associative clustering. Journal of Genetic Psychology, 1958, 92, 95-102.

Cole, M., Frankel, F., \& Sharp, D. Development of free recall learning in children. Developmental Psychology, 1971, 4, 109-123.

JABLonski, E. M. Free recall in children. Psychological Bulletin, 1974, 81, 522-539.

KoxuBun, $O$. The subjective organization in free recall learning by school children. Tohoku Psychological Folia, 1973, 32, 12-16.

LAURENCE, M. W. Age differences in performance and subjective organization in the free-recall learning of pictorial material. Canadian Journal of Psychology, 1966, 30, 388-399.

LiberTy, C., \& OrNstein, P. A. Age differences in organization and recall: The effects of training in categorization. Journal of Experimental Child Psychology, 1973, 15, 169-186.
Nelson, K. J. The organization of free recall by young children. Journal of Experimental Child Psychology, 1969, 8, 284-295.

Ornstein, P. A., Naus, M. J., \& LiberTy, C. Rehearsal and organizational processes in children's memory. Child Development, 1975, 46, 818-830.

Pellegrino, J. W. A general measure of organization in free recall for variable unit size and internal sequential consistency. Behavior Research Methods \& Instrumentation, 1971, 3, 241-246.

Roenker, D. L., Thompson, C. P., \& Brown, S. A. A comparison of measures for the estimation of clustering in free recall. Psychological Bulletin, 1971, 76, 45-48.

ROSNER, S. The effects of rehearsal and chunking instructions on children's multitrial free recall. Journal of Experimental Child Psychology, 1971, 11, 93-105.

ShAPIRo, S. I., \& Moely, B. Free recall, subjective organization, and learning to learn at three age levels. Psychonomic Science, 1971, 23, 189-191.

ThORNDIKE, E. L., \& LORGE, I. The teacher's word book of 30,000 words. New York: Teachers College, Columbia University, 1944.

Tulving, E. Subjective organization in free recall of "unrelated" words. Psychological Review, 1962, 69, 344-354.

(Received for publication August 19, 1976.) 